\title{
Enhanced astrocytic o-serine underlies synaptic damage after traumatic brain injury
}

\author{
Enmanuel J. Perez, ${ }^{1}$ Stephen A. Tapanes, ${ }^{1}$ Zachary B. Loris, ${ }^{1}$ Darrick T. Balu, ${ }^{2}$ Thomas J. Sick, ${ }^{3}$ Joseph T. Coyle, ${ }^{4}$ and Daniel J. Liebl \\ ${ }^{1}$ The Miami Project to Cure Paralysis, Department of Neurosurgery, University of Miami Miller School of Medicine, Miami, Florida, USA. ${ }^{2}$ Translational Psychiatry Laboratory, McLean Hospital, Belmont, \\ Massachusetts, USA. ${ }^{3}$ Department of Neurology, University of Miami Miller School of Medicine, Miami, Florida, USA. ${ }^{4}$ Laboratory of Psychiatric and Molecular Neuroscience, McLean Hospital, Belmont, \\ Massachusetts, USA.
}

\begin{abstract}
After traumatic brain injury (TBI), glial cells have both beneficial and deleterious roles in injury progression and recovery. However, few studies have examined the influence of reactive astrocytes in the tripartite synapse following TBI. Here, we have demonstrated that hippocampal synaptic damage caused by controlled cortical impact (CCI) injury in mice results in a switch from neuronal to astrocytic D-serine release. Under nonpathological conditions, o-serine functions as a neurotransmitter and coagonist for NMDA receptors and is involved in mediating synaptic plasticity. The phasic release of neuronal D-serine is important in maintaining synaptic function, and deficiencies lead to reductions in synaptic function and plasticity. Following $\mathrm{CCI}$ injury, hippocampal neurons downregulated o-serine levels, while astrocytes enhanced production and release of $\mathrm{D}$-serine. We further determined that this switch in the cellular source of D-serine, together with the release of basal levels of glutamate, contributes to synaptic damage and dysfunction. Astrocyte-specific elimination of the astrocytic D-serinesynthesizing enzyme serine racemase after $\mathrm{CCl}$ injury improved synaptic plasticity, brain oscillations, and learning behavior. We conclude that the enhanced tonic release of $\mathrm{D}$-serine from astrocytes after TBI underlies much of the synaptic damage associated with brain injury.
\end{abstract}

\section{Introduction}

Traumatic brain injury (TBI) is a devastating pathological condition that can lead to tissue loss and neurological dysfunction as a result of cell damage or death. Specialized membranes, such as at synapses, are susceptible to unique environmental changes within the injured brain, where astrocytes in the tripartite synapse contribute to synaptic transmission and function (1-3). TBI is known to result in astrocyte reactivity, which has both beneficial and deleterious effects on injury progression and recovery; however, few studies have examined the effects of astrogliosis on synaptic stability in the CNS (4-6).

In addition to direct damage to synaptic membranes, TBI can result in acute cell loss due to excessive release of excitatory amino acids such as glutamate (7-9). Glutamate excitotoxicity occurs when pathological levels of extracellular glutamate lead to enhanced extrasynaptic NMDAR activation, increased influx of calcium, enzymatic activation, and subsequent damage to cell structures $(8,10,11)$. NMDAR activation also requires the binding of a coagonist, such as D-serine or glycine. $\mathrm{D}$-serine is considered a more likely binding partner for NMDAR in the hippocampus, since its expression patterns match NMDAR expression (12-16) and it has a higher affinity for the NMDAR glycine-binding site (17).

Conflict of interest: J.T. Coyle is an inventor on US Patent Number 7,704,978 and four other related US patents, that are assigned to The General Hospital Corporation, Boston, Massachusetts, USA.

Submitted: December 14, 2016; Accepted: May 26, 2017.

Reference information: J Clin Invest. 2017;127(8):3114-3125.

https://doi.org/10.1172/JCI92300.
In the adult mammalian forebrain, $\mathrm{D}$-serine and its synthesizing enzyme, serine racemase (SRR), are expressed by excitatory glutamatergic neurons and subpopulations of GABAergic neurons $(12,18-20)$. Conversely, recent studies indicate that only a small percentage of astrocytes express a negligible amount of SRR in rodent forebrain tissues (21). Pathological levels of D-serine, similarly to glutamate, have been implicated in mediating acute excitotoxic cell death during pathological conditions (22-26); however, the effect of prolonged increases in $\mathrm{D}$-serine levels on synaptic function remains to be determined.

In the current study, we make use of a murine moderate controlled cortical impact (CCI) injury paradigm in which synaptic damage in the absence of neuronal cell death can model synaptic damage and recovery (27). We take advantage of inducible astrocyte- and neuron-specific Srr-knockout mice to examine the cellular contribution of $\mathrm{D}$-serine on synaptic stability and function in the tripartite synapse. Our findings demonstrate that CCI injury results in a switch in $\mathrm{D}$-serine release from neurons to astrocytes that contributes to synaptic damage and learning dysfunction associated with TBI.

\section{Results}

Phasic release of neuronal $D$-serine mediates synaptic transmission and long-term potentiation. To begin to evaluate the role of D-serine in regulating synaptic transmission, we first examined its expression using immunohistochemical approaches and function using electrophysiology in the naive hippocampus. D-serine was predominantly observed in the neuron-rich pyramidal cell (Figure 1, A and B) and granule cell layers, with low expression lev- 

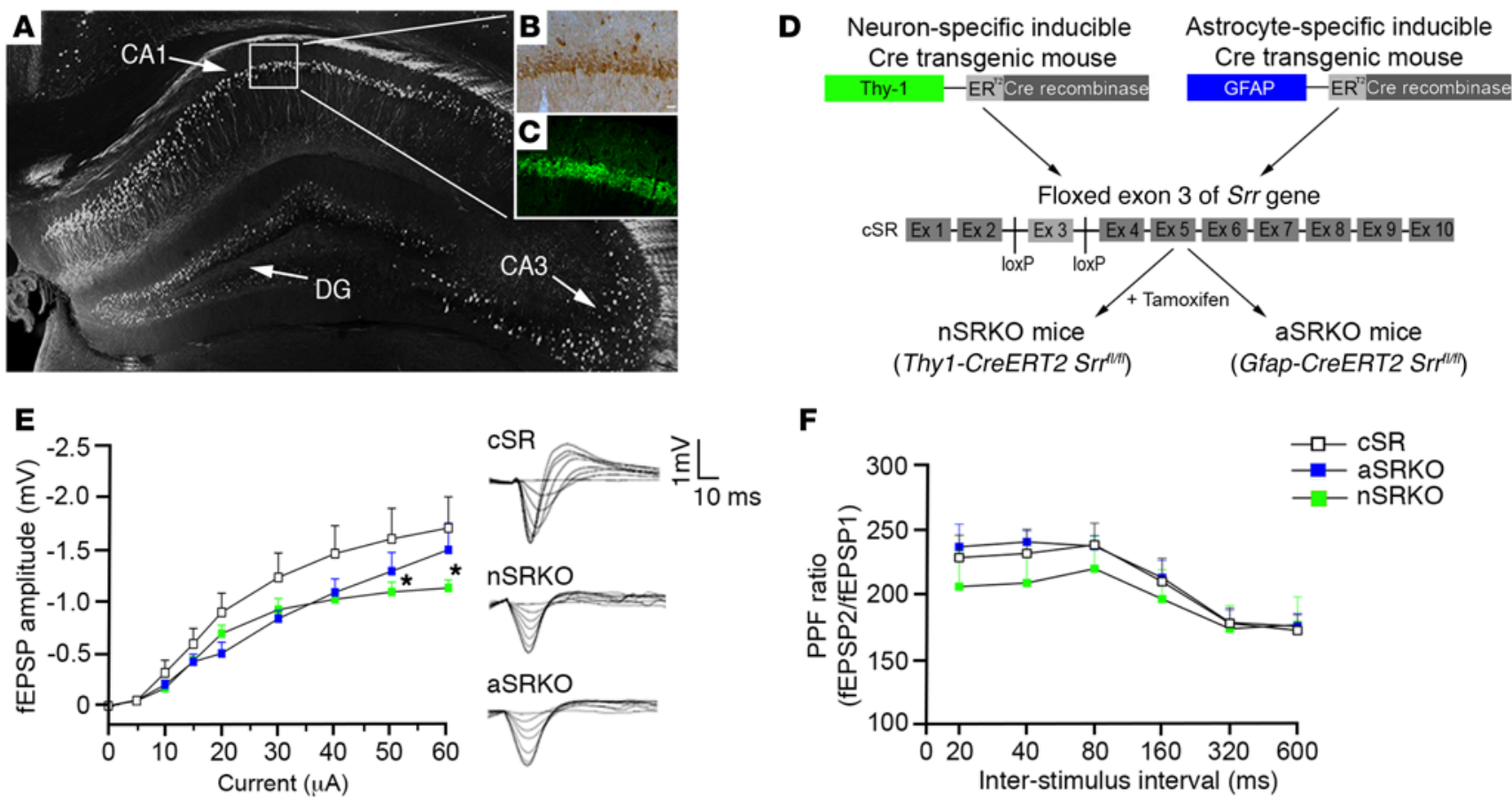

$\mathbf{F}$
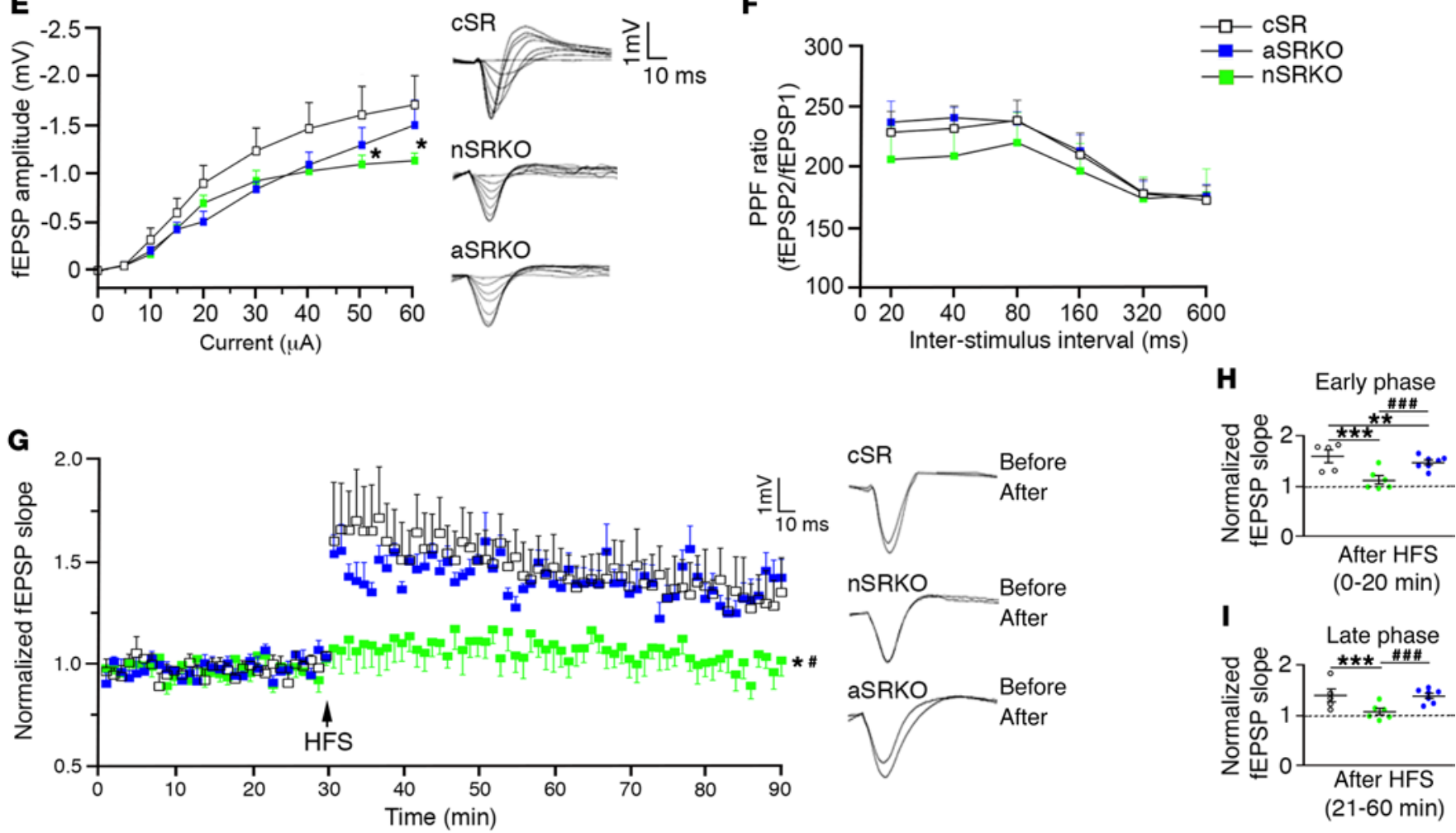

Figure 1. Neuronal D-serine is important for synaptic potentiation. D-serine (B) and SRR (C) immunoreactivity was observed in the CA1 pyramidal cell layer as outlined in a Thy-1-GFP-labeled hippocampus (A). Scale bar: $25 \mu \mathrm{m}$. (D) Schematic flow chart of the nSRKO mouse and the aSRKO mouse. (E) I/O curve showed reduced fEPSP amplitude for nSRKO mice at high intensities. Individual traces shown. (F) PPF showed no significant differences between genotypes. (G) LTP was reduced in nSRKO mice as compared with WT and aSRKO mice. Individual traces shown. (H) Histograph of LTP early phase showed reductions in nSRKO mice. (I) Histograph of LTP late phase showed reductions in nSRKO mice. Data represent mean \pm SEM. (E-I) $n=5-8 /$ group. (E-C) Two-way RM ANOVA. $(\mathrm{H}, \mathrm{I})$ One-way ANOVA. ${ }^{*} P<0.05 ;{ }^{* *} P<0.01 ;{ }^{* * *} P<0.001$, compared with cSR control mice. ${ }^{*} P<0.05$; ${ }^{\# \#} P<0.001$, compared with nSRKO mice.

els observed outside this zone. A similar expression profile was observed for the synthesizing enzyme SRR, essential for conversion of L-serine to D-serine (Figure 1, A and C). This expression profile supports previous studies showing that SRR and D-serine are primarily localized to neurons $(12,18-20)$.

To examine the contributions of neuronal and astrocytic D-serine, we examined local in vivo field potentials in the CA1 stratum radiatum in response to electrical stimulation of Schaffer collaterals in mice deficient for neuronal SRR (nSRKO; Thy1-CreERT2 Srft/fl mice) or astrocytic SRR (aSRKO; Gfap-CreERT2 Srr fl/ $^{\text {/f }}$ mice, where Gfap indicates glial fibrillary acidic protein) (Figure 1D and Supplemental Figure 1; supplemental material available online with this article; https://doi.org/10.1172/JCI92300DS1) as compared with control $\mathrm{Sr}^{A / / /}$ mice (cSR) that did not express Cre recombinase. SRR deficiencies are known to have a direct effect on $\mathrm{D}$-serine levels (12, 18). Following the delivery of an in vivo monophasic electrical pulse, input-output (I/O) curves indicated reduced field excitatory postsynaptic potentials (fEPSPs) in nSRKO mice, but only at high stimulating intensities (Figure 1E). These reductions suggest that neurons mainly contribute to the overall resting levels of $\mathrm{D}$-serine in the hippocampus, where activation of NMDAR is predominantly observed at higher stimulating intensities (28). No significant difference was observed in presynaptic function between genotypes using paired pulse facilitation (PPF) (Figure 1F). Potentiation of synaptic transmission following tetanic stimulation (i.e., long-term potentiation [LTP]) was only impaired in nSRKO mice, which showed a significant main effect for group $(P=0.0137)$ and time $(P<0.001)$ using 2-way ANOVA analysis (Figure 1G). Analysis of LTP induction and 

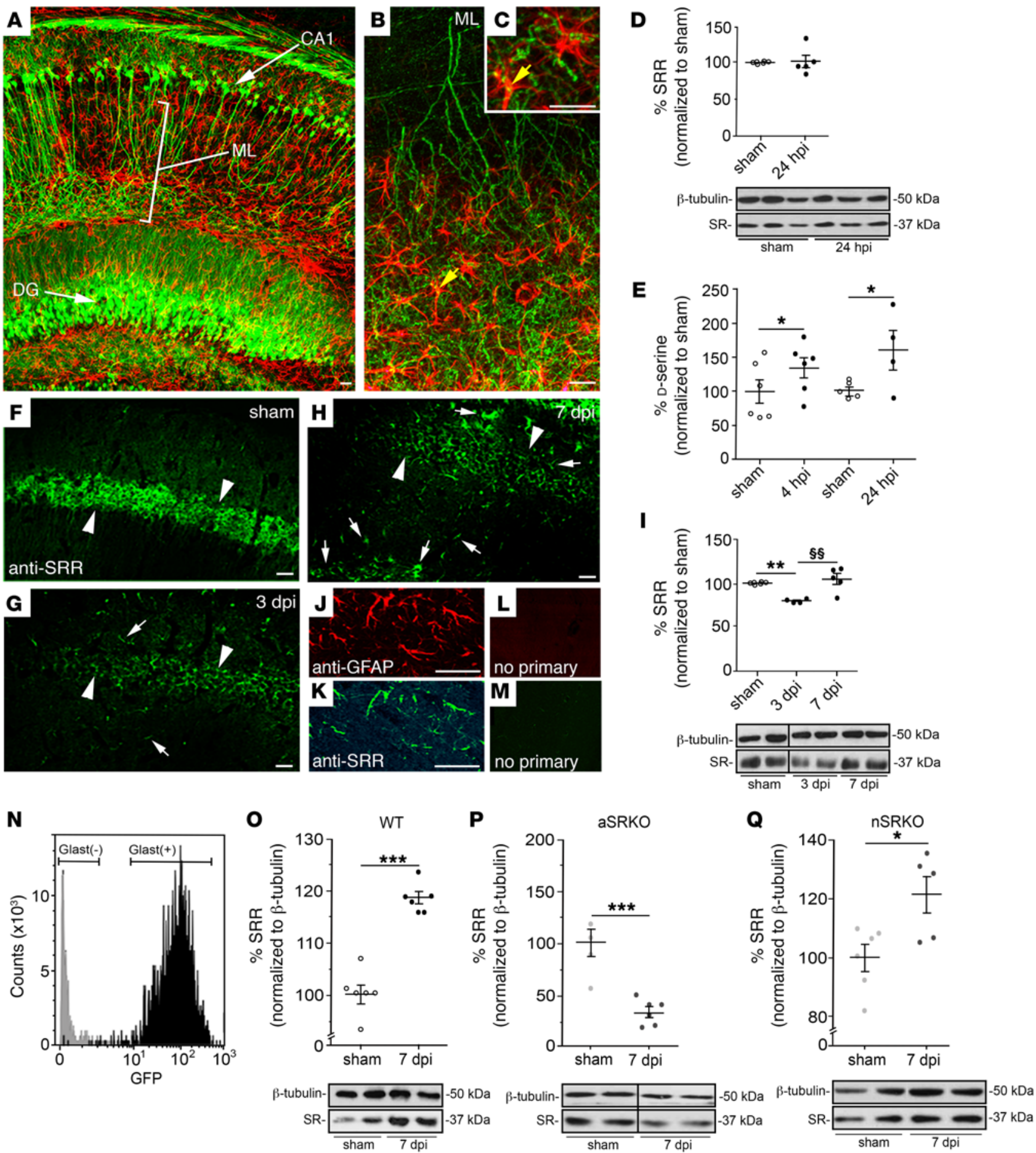

Figure 2. SRR is downregulated in neurons and upregulated in astrocytes after CCI injury. (A) CLARITY image of GFAP-labeled astrocytes (red) and GFP-expressing neurons (green) in Thy1-GFP transgenic mice shows close association of astrocytes and CA1 dendrites in the stratum lucidum/radiatum of the hippocampus. High-magnification images (B) and astrocyte-neuron membrane association (yellow; arrow) in stratum lucidum/radiatum (C). Scale bar: $25 \mu \mathrm{m}$. (D) SRR expression using Western blot analysis at $24 \mathrm{hpi}$ is not altered in the hippocampus. (E) D-serine levels using chemiluminescence analysis show increased levels at 4 and 24 hpi. (F) Immunohistochemical expression of SRR was reduced in pyramidal cell layer at 3 (G) and 7 (H) dpi, but increased in cells outside that region as compared with sham-treated controls (F). Scale bar: $25 \mu \mathrm{m}$. SRR-labeled cells outside the pyramidal cell layer (K) colabel with the astrocytic marker (J) GFAP. No crossreactivity observed in secondary only controls ( $\mathbf{L}$ and $\mathbf{M}$ ). Scale bar: $25 \mu \mathrm{m}$. (I) SRR levels were reduced at 3 dpi and returned to basal levels by 7 dpi using Western blot analysis. (N) Purification of hippocampal astrocytes from GFAP-GFP mice showed GFP-positive cells were only observed in isolated GLAST ${ }^{+}$isolated cells. (0) Isolated GLAST+ astrocytes express increased levels of SRR at 7 dpi using Western blot analysis. (P) SRR was reduced in the aSRKO hippocampus at $7 \mathrm{dpi}$ using Western blot analysis. (Q) SRR was increased in the nSRKO hippocampus at 7 dpi using Western blot analysis. Data represent mean \pm SEM. (D, E, I, O-Q) $n=4$-6/group. Student's $t$ test. ${ }^{*} P<0.05 ;{ }^{* *} P<0.01 ;{ }^{* *} P<0.001$, compared with sham-treated controls. ${ }^{\S} P<0.01$, compared with $3 \mathrm{dpi}$. For Western blot images, lanes were run on the same gel, but lanes separated by a line were noncontiguous (see Supplemental Figure 2). 
A

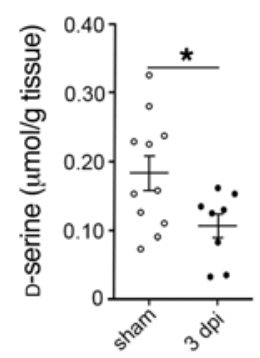

B
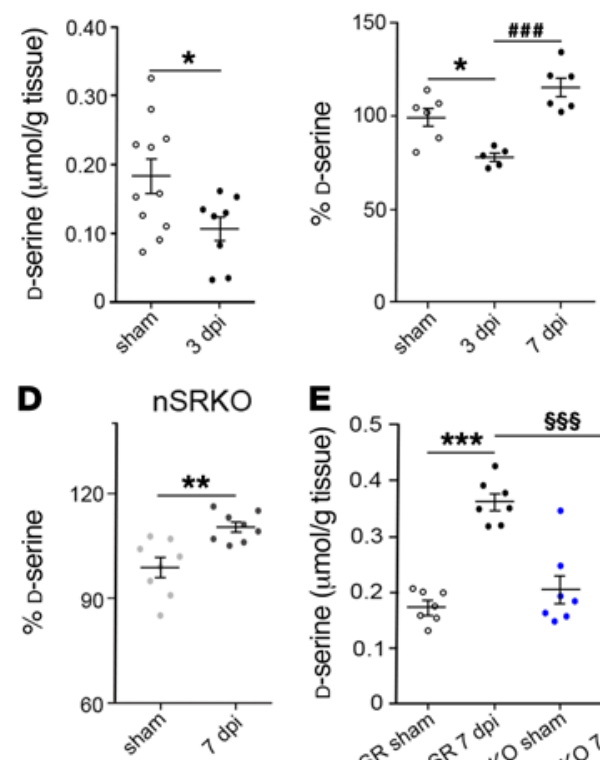

C Glast (+) WT astrocytes
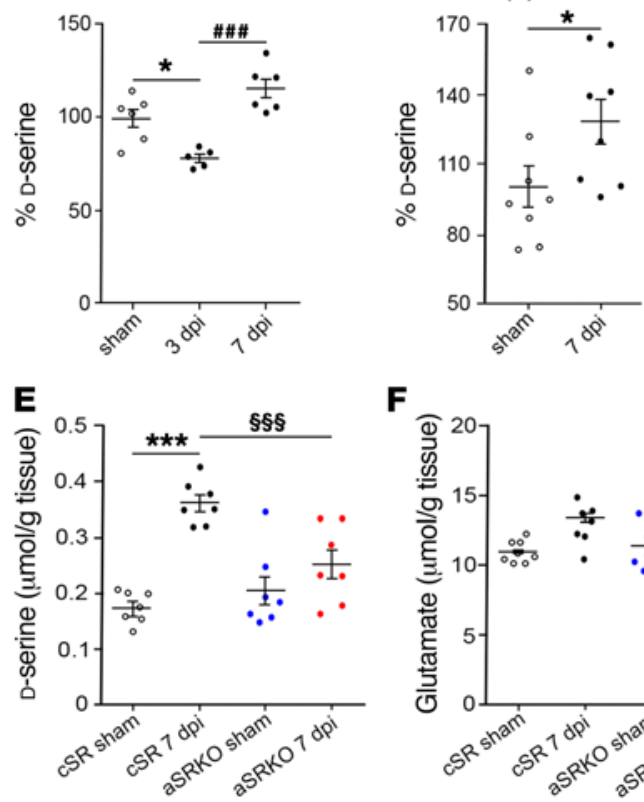

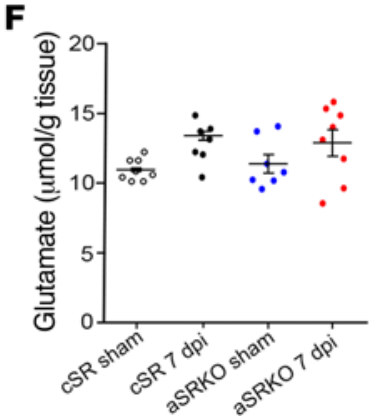

Figure 3. D-serine levels are increased in astrocytes at 7 dpi. (A) HPLC analysis shows reduced D-serine in the hippocampus at $3 \mathrm{dpi}$. (B) Chemiluminescence analysis showed reduced $\mathrm{D}$-serine at $3 \mathrm{dpi}$ that increases by $7 \mathrm{dpi}$. (C) Isolated GLAST ${ }^{+}$astrocytes have increased D-serine at $7 \mathrm{dpi}$. (D) D-serine levels remain increased in the nSRKO hippocampus. (E) HPLC analysis showed increased D-serine levels at $7 \mathrm{dpi}$ in CSR mice, but not sham-treated or CCI-injured aSRKO mice. (F) HPLC analysis showed no significant difference in glutamate levels in sham-treated or CCI-injured cSR or aSRKO mice at $7 \mathrm{dpi}$. Data represent mean $\pm \mathrm{SEM}$. (A) $n=8-11 /$ group; (B, E, F) $n=6-8 /$ group; (C and D) $n=6 /$ group repeated in triplicate. (A-D) Student's $t$ test. (E and $\mathbf{F}$ ) One-way ANOVA. ${ }^{*} P<0.05$; ${ }^{* *} P<0.01 ;{ }^{* *} P<0.001$, compared with sham-treated controls. ${ }^{\# \#} P<0.001$, compared with CSR at $7 \mathrm{dpi}$. ${ }^{\$ \S} P<0.001$, compared with $3 \mathrm{dpi}$. maintenance showed significant reduction in both early $(P<0.001)$ and late-phase $(P<0.001)$ LTP in nSRKO mice as compared with cSR and aSRKO sham-treated mice (Figure 1, $\mathrm{H}$ and I). These in vivo observations strongly support a predominant role for phasic release of neuronal D-serine in synaptic plasticity.

CCI injury leads to a cellular switch in SRR expression in the hippocampus. Astrocytosis is known to result in a spectrum of morphological and molecular changes that can influence tissue pathology; however, the effects of reactive astrocytes on synapse function after TBI remain poorly resolved. In the hippocampal tripartite synapse, CLARITY imaging showed close interactions of astrocytes with synaptic membranes originating from CA1 pyramidal neurons (CA1) (Figure 2, A-C), CA3 pyramidal neurons (CA3), and dentate granule (DG) cells. To examine the role of $\mathrm{D}$-serine in the traumatic injured brain, we had recently developed a CCI injury model that resulted in synaptic damage in the absence of hippocampal neuron cell death (27). This model enables us to examine the mechanisms of synaptic damage independently of neuronal loss.

In the CCI-injured hippocampus, Western blot analysis showed little change in total SRR levels at 24 hours post CCI injury (hpi) as compared with those in the sham-treated controls (Figure 2D and Supplemental Figure 2), but chemiluminescence analysis of total D-serine levels showed a significant increase at 4 and 24 hpi (Figure 2E). This increase in D-serine likely represents an acute injury-induced conversion of L- to D-serine in neurons. At 3 and 7 days post-CCI injury (dpi), SRR expression patterns and levels changed dramatically (Figure 2, F-H). SRR expression was visibly decreased, by more than $50 \%$, in the hippocampal pyramidal cell layer (Figure 2, G and H; arrowheads) as compared with sham-treated controls (Figure $2 \mathrm{~F}$ ). Western blot analysis showed an approximately $25 \%$ decrease $(P<0.01)$ in total SRR levels at $3 \mathrm{dpi}$ (Figure 2I). This discrepancy most likely is the result of increased SRR expression in astrocytes outside the pyramidal cell layer, including regions of the stratum oriens and lucidum/ radiatum (Figure 2G; arrows). At $7 \mathrm{dpi}$, expression in the pyra- midal cell layer remained low (Figure 2H; arrowheads), while greater numbers of glial cells expressed SRR (Figure 2H; arrows). Western blot analysis showed that SRR expression returned to preinjury levels by $7 \mathrm{dpi}$, representing a significant increase $(P<0.01)$ from 3 dpi (Figure 2I). Confocal imaging demonstrated that the majority of SRR-positive cells outside the pyramidal cell layer colocalized with GFAP-positive astrocytes (Figure 2, J and $\mathrm{K})$, which was not observed in controls using only the secondary antibody (Figure 2, L and M).

To confirm that SRR protein levels were increased specifically in astrocytes after CCI injury, we column purified ( $>98 \%$ pure) astrocytes using glutamate-aspartate transporter (GLAST) antibodies (Figure $2 \mathrm{~N}$ and Supplemental Figure 4 ) followed by Western blot analysis for SRR. We observed a $21 \%$ increase $(P<0.001)$ in SRR levels at $7 \mathrm{dpi}$ in astrocytes isolated from the CCI-injured hippocampus as compared with sham-treated controls (Figure 2O). SRR levels at 7 dpi in the aSRKO whole hippocampus were reduced $(P<0.001)$ compared with those in sham-treated controls (Figure 2P), lending greater support for switch in the cellular expression pattern of SRR after CCI injury. In whole hippocampal tissues isolated from nSRKO mice (Figure 2Q), where SRR levels had been shown to be reduced in neurons at $7 \mathrm{dpi}$ (Figure $2 \mathrm{H}$ ), we observed an increase similar to that observed in WT mice (Figure 2O). Taken together, these findings support our conclusion that TBI results in a downregulation of neuronal SRR and upregulation of astrocytic SRR levels within the first week after injury.

CCI injury increases astrocyte-derived D-serine levels. To demonstrate that increased astrocytic SRR expression leads to an increase in $\mathrm{D}$-serine synthesis, we examined hippocampal $\mathrm{D}$-serine in the presence and absence of astrocyte-derived SRR (Figure 3). Similarly to what occurred with SRR levels, we observed a significant reduction in D-serine levels at $3 \mathrm{dpi}$ in WT mice using HPLC (0.108 $\pm 0.018 \mu \mathrm{mol} / \mathrm{g}$ tissue; $P<0.05)$ as compared with sham-treated controls (0.184 $\pm 0.025 \mu \mathrm{mol} / \mathrm{g}$ tissue) (Figure $3 \mathrm{~A})$. Chemiluminescent assay of D-serine supported our HPLC observations at $3 \mathrm{dpi}$, 
and by $7 \mathrm{dpi}$, D-serine levels were significantly increased $(P<0.001)$ as compared with those at $3 \mathrm{dpi}$ (Figure $3 \mathrm{~B}$ ). Examination of isolated astrocytes from WT mice showed a $20 \%$ increase $(P<0.05)$ in $D$-serine levels at $7 \mathrm{dpi}$ (Figure $3 \mathrm{C}$ ), which was similar to whole hippocampal D-serine levels $(P<0.01)$ observed in CCI-injured nSRKO mice compared with sham-treated controls (Figure 3D). We next compared aSRKO mice with cSR control mice (Figure 2B), where both received tamoxifen (TAM) treatment 2 weeks prior to injury. In cSR mice, HPLC analysis showed a 2-fold increase $(0.363 \pm 0.015$ $\mu \mathrm{mol} / \mathrm{g}$ tissue; $P<0.001)$ in hippocampal $\mathrm{D}$-serine levels at $7 \mathrm{dpi}$ as compared with cSR sham-treated $(0.176 \pm 0.011 \mu \mathrm{mol} / \mathrm{g}$ tissue $)$ mice (Figure 3E). In aSRKO mice, no significant differences were observed between sham-treated $(0.206 \pm 0.026 \mu \mathrm{mol} / \mathrm{g}$ tissue $)$ and CCI-injured $(0.253 \pm 0.026 \mu \mathrm{mol} / \mathrm{g}$ tissue $)$ mice, whereas CCIinjured aSRKO mouse D-serine levels were significantly $(P<0.01)$ less than levels of CCI-injured cSR mice (Figure 3E). This was further supported by examination of Srr mRNA in aSRKO tissues, where Srr mRNA levels were similar between sham-treated and CCI-injured mice in 7 dpi WT tissues, but reduced in CCI-injured aSRKO mice (Supplemental Figure 3). No significant changes were observed in amino acid oxidase mRNA levels, such as D-amino acid oxidase (DAO) and amine oxidase, copper-containing 1 (AOC1) enzymes, between WT and aSRKO sham-treated mice and 7 dpi tissues (Supplemental Figure 3), suggesting that changes in SRR and not large reductions in D-serine-metabolizing enzymes underlie CCI injury-induced increases in D-serine. Taken together, our observations confirm that CCI injury results in a switch in SRR (Figure 2) and D-serine (Figure 3) expression in the hippocampus from neurons to astrocytes over the first week after injury.

Since glutamate serves as the NMDAR agonist, glutamate levels were examined in CCI-injured mice to determine whether its expression was altered in aSRKO mice. HPLC analysis showed no significant differences in glutamate levels between groups or after CCI injury (cSR sham, $10.96 \pm 0.31 \mu \mathrm{mol} / \mathrm{g}$ tissue; cSR CCI, $13.42 \pm 0.71 \mu \mathrm{mol} / \mathrm{g}$ tissue; aSRKO sham, $11.41 \pm 0.67$ $\mu \mathrm{mol} / \mathrm{g}$ tissue; aSRKO CCI, $12.87 \pm 0.93 \mu \mathrm{mol} / \mathrm{g}$ tissue) (Figure $3 \mathrm{~F}$ ), which is similar to what we have previously reported for WT mice at periods between 4 hpi and 7 dpi (27). Although our findings suggest that CCI injury or alterations in $\mathrm{D}$-serine expression do not change total glutamate levels, we cannot exclude the possibility that glutamate homeostasis may be affected during the first week after injury.

Reducing astrocytic D-serine spares the hippocampus from CCI injury-induced dysfunction. We next determined whether inhibiting astrocytes from producing D-serine (i.e., aSRKO mice) altered synaptic transmission and plasticity after CCI injury when reactive astrocytes appeared. Consistent with our observations using WT mice (27), CCI injury in cSR control mice led to a significant downward shift in fEPSP amplitude $(P<0.01)$ at higher stimulus intensities $(40-60 \mu \mathrm{A})$ as compared with cSR and aSRKO sham-treated controls (Figure 4A). These previous studies had shown that synaptic damage and losses and not neuronal loss from CCI injury underlie the observed deficits in synaptic transmission and plasticity (27). Similar trends were also observed in PPF between groups, where the PPF ratio was attenuated in CCI-injured cSR mice at shorter interstimulus intervals as compared with cSR and aSRKO controls (Figure 4B). Interestingly, we did not observe a significant reduction in the I/O curve or PPF in CCI-injured aSRKO mice, although there was a downward shift in PPF ratio curve compared with sham-treated controls.

We next evaluated synaptic plasticity in the presence and absence of astrocytic D-serine. Two-way repeated measures (RM) ANOVA of normalized fEPSP after high-frequency stimulation (HFS) showed significant interaction $(P<0.001)$ as well as a significant main effect for animal groups $(P=0.0203)$ and time $(P<0.001)$. In vivo HFS of sham-treated cSR mice resulted in LTP that was not observed in CCI-injured cSR mice (Figure 4C). Conversely, aSRKO sham-treated and CCI-injured mice both had a significant synaptic potentiation following in vivo tetanic stimulation (Figure 4C), where CCI-injured aSRKO mice had significantly greater potentiation $(P<0.05)$ than CCI-injured cSR mice. Our results suggest that astrocyte-derived $D$-serine plays a substantial role in the synaptic dysfunction observed following CCI injury. Analysis of LTP induction and maintenance in CCI-injured cSR mice showed a large reduction in both early $(P<0.001)$ and late-phase $(P<0.001)$ LTP as compared with sham-treated cSR mice (Figure 4, D and E). A smaller reduction in early phase LTP was observed in sham-treated and CCI-injured aSRKO mice; however, this was not maintained in the late LTP phase. Comparison of late-phase LTP between CCI-injured cSR and aSRKO mice showed significantly higher potentiation $(P<0.001)$ in mice deficient in astrocyte-derived SRR. Together, these findings demonstrate that the release of $\mathrm{D}$-serine from astrocytes underlies TBI-induced synaptic dysfunction and that blocking this D-serine source is neuroprotective.

Examination of hippocampal field potential oscillations in vivo in CCI-injured mice showed a significant interaction between frequencies and experimental groups using 2-way ANOVA analysis $(P=0.002)$. Examination of $\theta$ oscillations at the $4-$ to $5-\mathrm{Hz}$ frequency range showed significantly greater $(P<0.001)$ power in CCI-injured cSR mice as compared with that in all the other groups (Figure 4F). Additionally, power at the 4 - to $5-\mathrm{Hz}$ range in CCI-injured aSRKO mice was substantially reduced as compared with that in CCI-injured cSR mice at $7 \mathrm{dpi}$. Our results suggest that CCI injury leads to an enhancement in $\theta$ rhythms, which likely involves D-serine release and activation of NMDARs.

To verify whether our observed physiological effects translate into behavioral responses, we examined hippocampal-associated learning and memory using a fear-conditioning paradigm (Figure 4, G and H). Following CCI injury, all groups were habituated at $5 \mathrm{dpi}$, trained at $6 \mathrm{dpi}$, and tested at $7 \mathrm{dpi}$. No significant differences were observed in freezing behavior during the training periods, while significant interactions and main effects were observed among groups $(P=0.0084)$ and on test days compared with training days $(P<0.001)$. CCI injury resulted in an approximately $50 \%$ reduction in contextual (Figure $4 \mathrm{G}$ ) and cue (Figure $4 \mathrm{H}$ ) freezing behavior in CCI-injured cSR mice as compared with in sham-treated cSR mice. Contextual freezing behavior was significantly increased $(P<0.05)$ in sham-treated aSRKO mice as compared with that in sham-treated cSR mice, and although the basis for this enhanced freezing response is unclear, it may result from other limbic structures not examined in our analysis. What is clear is that CCI-injured aSRKO mice were not significantly different from sham-treated aSRKO mice (Figure 4G). Comparison 

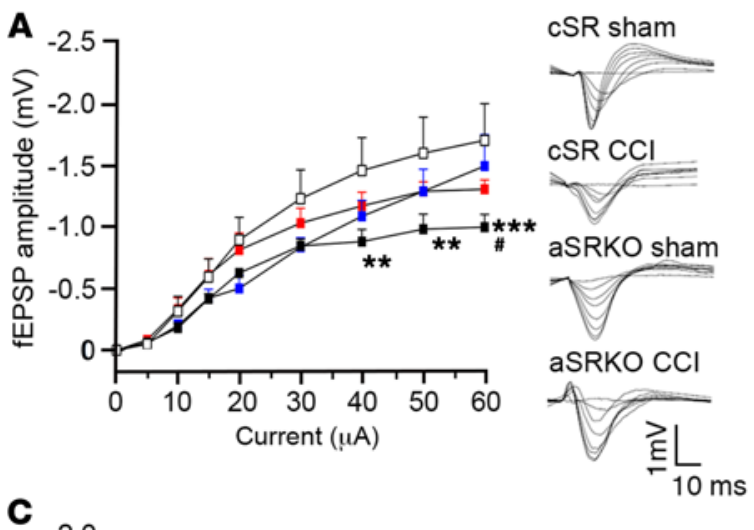

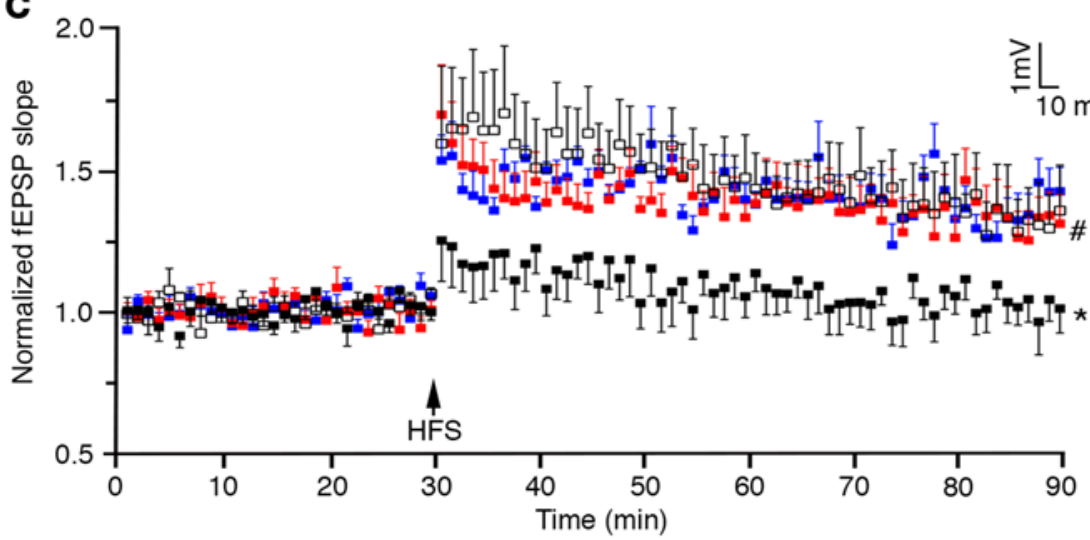

$\mathbf{F}$

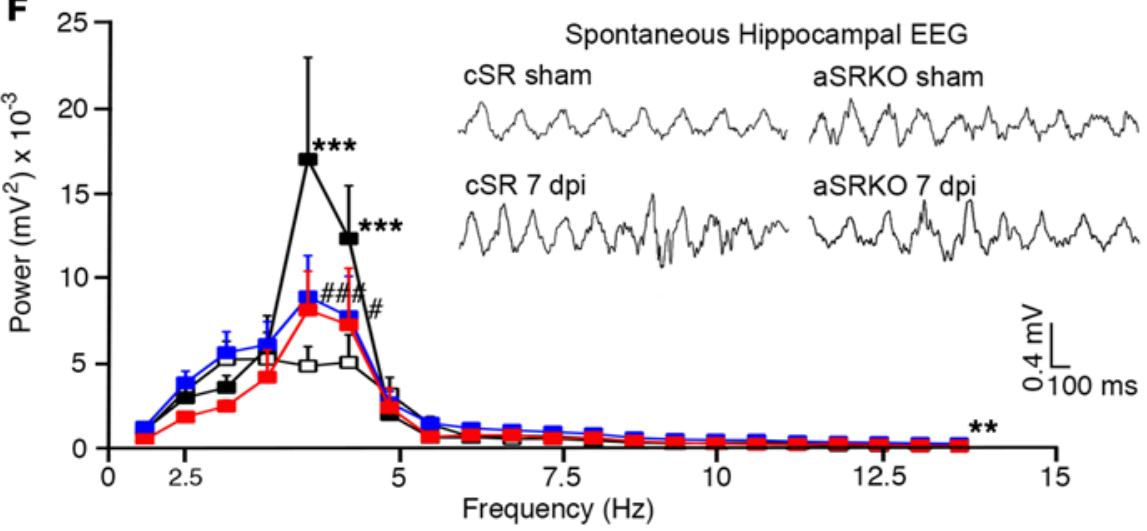

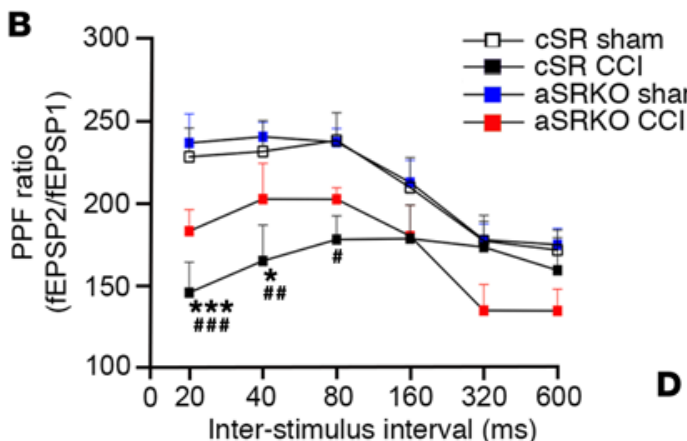
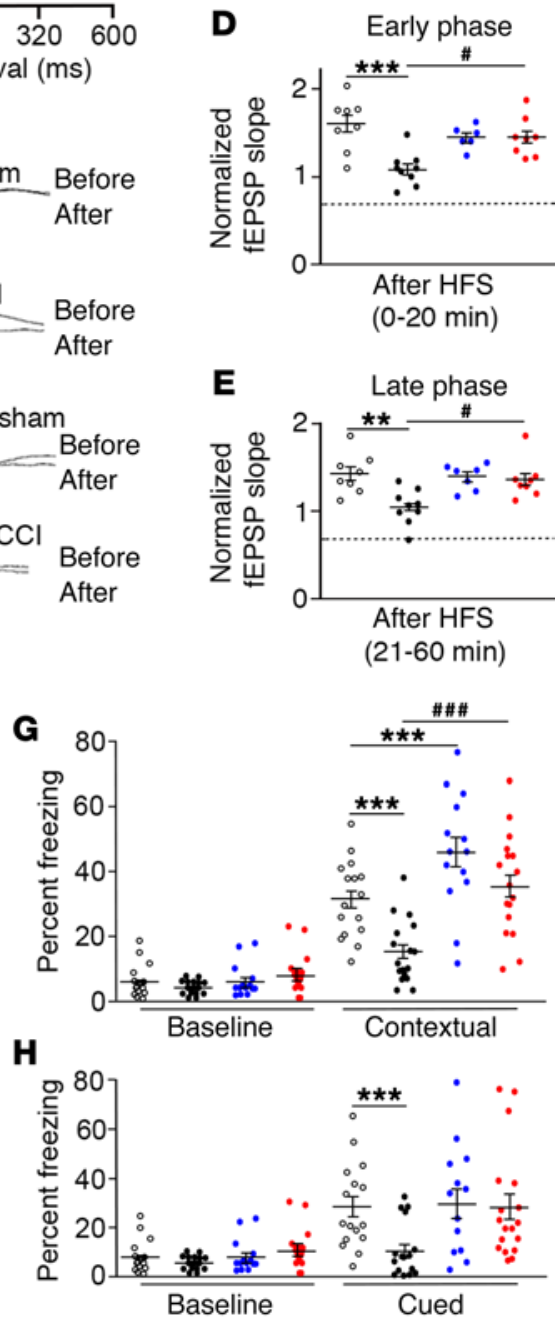

Figure 4. Synaptic dysfunction and learning deficits are not observed in aSRKO CCI-injured mice at $7 \mathrm{dpi}$. (A) I/O curve showed reduced fEPSP amplitude at high intensities for CSR CCI-injured mice as compared with sham-treated and CCI-injured aSRKO mice. Individual traces shown. (B) PPF showed a reduced ratio for CSR CCI-injured mice as compared with sham-treated and CCI-injured aSRKO mice. (C) LTP was reduced in CCI-injured cSR control mice, but not sham-treated CSR, sham-treated aSRKO, or CCI-injured aSRKO mice. Individual traces shown. (D) Histograph of LTP early phase showed greatest reductions in CCI-injured cSR mice and reductions to a lesser extent in aSRKO mice. (E) Histograph of LTP late phase showed greatest reductions in CCI-injured cSR mice and reductions to a lesser extent in aSRKO mice. Early and late phase slopes of CCI-injured aSRKO mice were significantly greater than CCI-injured cSR mice at $7 \mathrm{dpi}$. (F) $\theta$ rhythm frequency was increased in CCI-injured cSR mice and was increased to a lesser extent in sham-treated and CCl-injured aSRKO mice at frequencies between 4 and $5 \mathrm{~Hz}$ as compared to sham-treated cSR mice. $\theta$ rhythm frequency of CCI-injured aSRKO mice were significantly less than CCI-injured cSR mice. $\theta$ rhythm traces were shown for amplitude versus time. Fear-conditioning tests showed significantly decreased freezing behavior in CCI-injured CSR mice for contextual (C) and cued (H) learning that was not observed in sham-treated or CCI-injured aSRKO mice. Data represent mean \pm SEM. (A-F) $n=7-10$ /group. (G and $\mathbf{H}) n=16-18 /$ group. $(\mathbf{A}-\mathbf{C}, \mathbf{F}-\mathbf{H})$ Two-way RM ANOVA. (D and E) One-way ANOVA. ${ }^{*} P<0.05$, ${ }^{* *} P<0.01$; ${ }^{* *} P<0.001$, compared with cSR control mice. ${ }^{\#} P<0.05$; ${ }^{\#} P<0.01 ;{ }^{\# \#} P<0.001$, compared with cSR at 7 dpi.

of CCI-injured aSRKO mice with CCI-injured cSR mice showed significantly higher $(P<0.001)$ contextual freezing behavior in the former. Similar but nonsignificant trends $(P=0.0678)$ between CCI-injured cSR and aSRKO mice were observed in cued freez- ing behavior. These behavioral studies support our observations of synaptic plasticity and together suggest that astrocyte-derived D-serine likely underlies the synaptic dysfunction and/or damage associated with reduced synaptic plasticity. 

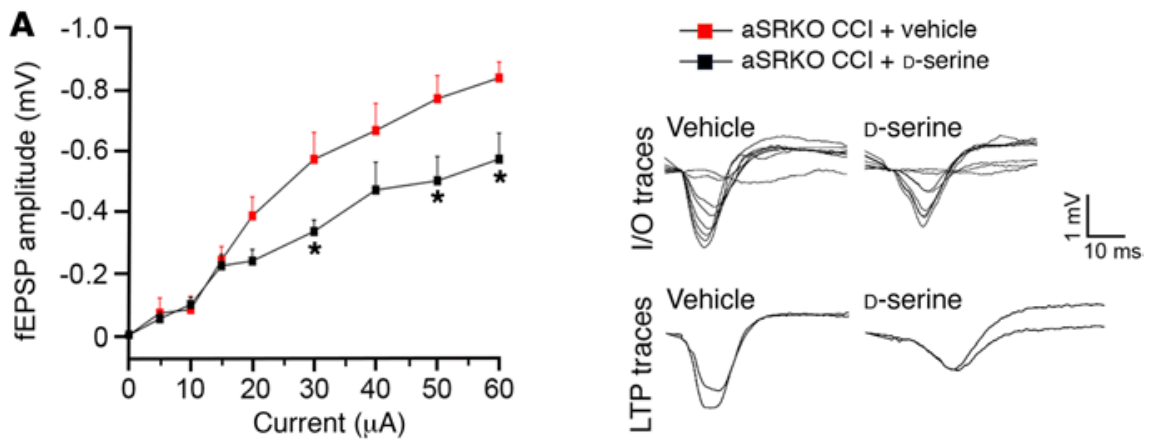

Figure 5. D-serine administration led to synaptic dysfunction in aSRKO mice at $7 \mathrm{dpi}$. (A) I/O curve showed reduced fEPSP slopes in $840 \mu \mathrm{g} / \mathrm{kg} / \mathrm{d}$ o-serine-treated, CCl-injured aSRKO mice as compared with those in vehicle-treated aSRKO mice. Individual traces shown. (B) LTP is reduced in D-serine-treated CCI-injured aSRKO mice as compared with that in vehicle-treated aSRKO mice. Individual traces shown. Data represent mean \pm SEM. (A and B) $n=4$ /group. Two-way RM ANOVA. ${ }^{*} P<0.05$; ${ }^{* *} P<0.001$, compared with vehicle-treated, CCI-injured aSRKO mice.

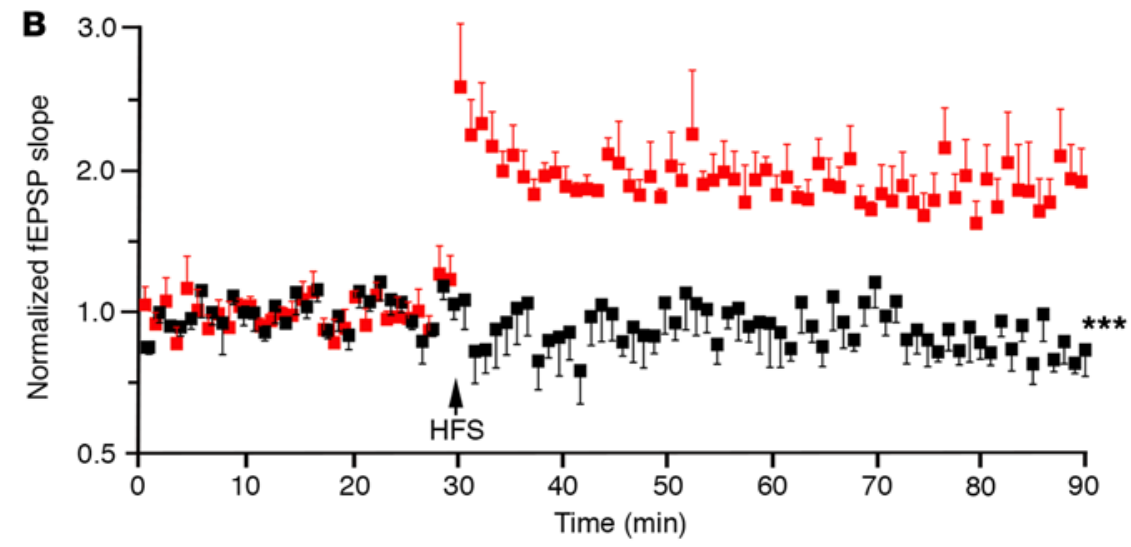

Increasing D-serine can reverse the enhanced synaptic potentiation observed in aSRKO mice after CCI injury. To validate our conclusion that CCI injury-induced synaptic dysfunction is aggravated by increased D-serine levels observed in the CCI-injured aSRKO mice, we examined CA3-CA1 hippocampal fEPSPs in vivo in CCI-injured aSRKO mice after 7-day infusion of vehicle or D-serine $(840 \mu \mathrm{g} / \mathrm{kg} / \mathrm{d})$ into the contralateral lateral ventricle (Figure 5). D-serine infusion into CCI-injured aSRKO mice resulted in reduced basal synaptic transmission as revealed by a downward shifted I/O curve at stimulation intensities of $30 \mu \mathrm{A}$ or higher compared with that in vehicle-infused controls (Figure 5A). No significant differences were observed in PPF between groups (not shown); however, D-serine infusion reversed the significant potentiation after HFS observed in aSRKO CCI-injured mice (Figure 5B) to levels similar to those observed in CCI-injured cSR mice (Figure 4C). These findings demonstrate that high levels of $\mathrm{D}$-serine in the injured hippocampus are detrimental to synaptic transmission and plasticity.

\section{Discussion}

TBI is a dynamic and progressive disorder that can lead to the disruption of brain circuitry that underlies many of the functional deficits observed in TBI patients. One component prevalent in all brain injuries, ranging from mild to severe, is synaptic damage. In regions where synaptic plasticity is important for cognitive function, glial cells play critical roles in modulating synaptic transmission and plasticity. In fact, several proteins have been implicated in glial regulation of synaptic functions after TBI, such as matrix metalloproteinases, astrocytic signal transducers and activators of transcription, and the calcineurin-dependent transcription factor nuclear factor of activated T cells (NFAT) (4-6). Here, we show that astrocytes in the tripartite synapse are active participants in promoting synaptic damage following CCI injury, where enhanced D-serine release leads to slow excitotoxic synaptic damage in the hippocampus. In nonpathological conditions, D-serine is produced and released by neurons to regulate the resting state of NMDAR activation. In these conditions, a phasic release of $\mathrm{D}$-serine by neurons elicits LTP. CCI injury results in reduced synaptic transmission, plasticity, synchrony, and memory as a result of increased astrocytic D-serine release. Astrocyte-specific gene-targeted deletion of Srr restored synaptic responses and learning, which can be reverted back to injury states by $\mathrm{D}$-serine administration.

In nonpathological conditions, glutamate release can activate both $\alpha$-amino-3-hydroxy-5-methyl-4-isoxazolepropionic acid receptors (AMPARs) and NMDARs, where NMDARs play a regulatory role in basal synaptic transmission $(29,30)$. Unlike AMPARs, NMDARs require multiple inputs, including binding of the coagonist $\mathrm{D}$-serine and $\mathrm{Ca}^{+2}$ permeation, which are essential components of long-lasting alterations in synaptic strength. A monophasic electrical pulse can activate AMPARs at low intensities; however, high intensities are known to activate both AMPARs and NMDARs (28). HFS might selectively increase D-serine levels by stimulating release from the neuronal pool, which is reduced in neuronal SRR-deficient mice, leading to deficits in synaptic plasticity. These findings are supported by studies in which stimulating D-serine release from neurons enhanced LTP in mouse and rat hippocampal slices (31) and neuron-specific deletion of the $\operatorname{Srr}$ gene led to reduced spine density and dendritic arborization (32) as well as LTP deficits (14). To date, several groups have confirmed that SRR is expressed by inhibitory and excitatory neurons throughout the mammalian brain $(12,19,33)$, where neuronal $\mathrm{D}$-serine is mainly released in response to depolarization (20). 
An important component of our study was to employ a murine model of synaptic damage in the absence of neuronal loss in order to directly evaluate the mechanisms that regulate synaptic dynamics within the complex and evolving TBI environment. Our CCI injury model produces a moderate cortical injury with only mild damage to subcortical tissues, such as the hippocampus, where synaptic damage and loss within the hippocampus underlie learning and memory deficits (27). This enabled us to examine discrete and reversible synaptic responses while maintaining the overall environmental characteristics associated with TBI, including distal tissue losses (34), reactive gliosis (27), a compromised blood-brain barrier, and inflammatory responses (35). The presence of astrocytosis in the CCI-injured hippocampus allowed us to examine how gliotransmitters from reactive astrocytes may regulate synaptic function and/or dysfunction. It has been well documented that astrocytes release glutamate minutes following TBI $(8,36)$, which leads to an acute activation of NMDARs and AMPARs, excessive $\mathrm{Ca}^{+2}$ influx, and, ultimately, cell death in severely compromised tissues (i.e., glutamate excitotoxicity). NMDARs are also involved in slow excitotoxicity that occurs over hours and days after injury and have been associated with enhanced D-serine levels $(37,38)$. While we cannot exclude the possibility that $\mathrm{D}$-serine contributes to excitotoxic cell death in regions of severe injury, our studies do support a direct role for astrocytic D-serine in slow excitotoxic synaptic damage in the CCI-injured hippocampus. What is less clear is whether increased astrocytic D-serine is sufficient to induce synaptic damage independently of neuronal D-serine reductions that occur after CCI injury. Importantly, our findings in astrocytic SRR-deficient mice, where synaptic plasticity and learning behaviors are restored to preinjury levels, support this possibility. We also cannot exclude the possibility that glutamate participates in D-serine-mediated slow excitotoxicity. Total hippocampal glutamate levels are not altered between 4 hours and $7 \mathrm{dpi}$ (27), but potential alterations in glutamate release and reuptake may participate in our observed delayed synaptic damage. Overall, it is clear that astrocytic D-serine release is a key player in mediating synaptic damage after CCI injury.

Learning involves an intricate interplay of a multitude of neural circuits in response to an experience. Within the hippocampus, $\theta$ oscillations are known to facilitate memory processes, although the exact mechanisms are not completely understood. TBI can result in a decrease in power in the $\theta$ frequency band $(39,40)$. However, changes in $\theta$ frequency after TBI are dependent on the severity of the injury (41). It is important to note that hippocampal $\theta$ rhythms occur at different frequencies dependent on the state of wakefulness of the animal. The higher frequency rhythm (7-9 $\mathrm{Hz}$ ) occurs when animals are exploring and has been termed type 1, while the slower type 2 rhythm $(3-5 \mathrm{~Hz})$ occurs when animals are in a quiescent state of sensory processing (42). Only type 2 $\theta$ is observed in animals under urethane anesthesia (43), which was used in the present study. Thus, the increase in hippocampal field oscillation power observed here is the first report, to our knowledge, of the effect of TBI on type $2 \theta$, as other studies of TBI were conducted in awake, freely moving animals. Curiously, progressive cognitive dysfunction, such as in Alzheimer's disease (AD) or Parkinson's disease (PD) models, has been associated with an increase in hippocampal $\theta$ power at rest as opposed to a decrease in $\theta$ power after severe TBI $(44,45)$. In this regard, our mild-to-moderate CCI model involving synaptic damage without neuronal losses is more akin to models of progressive synaptic dysfunction, such as AD models, where injury results in a marked increase in $\theta$ power associated with increased astrocytic D-serine levels. Alternatively, impaired NMDAR activation on parvalbumin-positive interneurons can result in the changes observed (44). Interestingly, whereas NMDAR hypofunction impairs hippocampal $\theta$ oscillations, increased activation of neuronal NMDARs due to increased availability of D-serine after injury can potentially enhance $\theta$ oscillations (46). Augmentation of NMDAR activity can also result from excitatory feedback to neurons through gliotransmitters modifying NMDAR-mediated currents (46-48). In short, we show a role for glial-derived D-serine in both LTP and $\theta$ oscillations in the injured hippocampus.

The role of synaptic and extrasynaptic receptors in neuronal cell death is currently a matter of intense debate, where excitotoxicity is thought to involve extrasynaptic NMDARs (36, 49-51). We did not determine whether reactive astrocytic $\mathrm{D}$-serine activates synaptic and/or extrasynaptic NMDARs after CCI injury, but we have shown that the NMDAR GluN2B subunit is elevated in our CCI injury model (27). GluN2B subunits are known to segregate outside the synapses, whereas GluN2A subunits are confined to the postsynaptic membrane $(11,25,52,53)$. Previously, D-serine has been shown to preferentially bind GluN1/GluN2A-containing NMDARs in synaptic sites, whereas glycine may preferentially bind GluN1/GluN2B-containing receptors in the extrasynapse (54). However, coagonist binding with a particular NMDAR subunit is not a strict rule. NMDAR heterotrimers can also exist, containing GluN1 and both GluN2A and GluN2B subunits (30, $55)$. At this time, the synaptic segregation of NMDAR subunits in our CCI injury model is unclear and additional studies are needed to determine whether enhanced astrocytic D-serine activates GluN2B-containing extrasynaptic NMDARs.

In summary, our findings demonstrate that excessive release of the gliotransmitter D-serine from reactive astrocytes may regulate slow excitotoxic damage to the brain's circuitry that underlies some of the cognitive dysfunction associated with TBI. Further advances on the role of gliotransmission in regulating synaptic stability, function, and regeneration will provide the foundation for developing therapeutic strategies to prevent TBI neuronal damage and enhance TBI patient recovery.

\section{Methods}

Animals. The generation of mutant mice involved breeding mice in which the first coding exon of SRR had been flanked with loxP sites $\left(\mathrm{Sr}^{\mathrm{f} / \mathrm{fl})}\right)$ as previously described $(18,56)$, bred with either Thy1CreERT2 mice (JAX Mice, Jackson Laboratory, 012708; Tg[Thy1-cre/ ERT2,-EYFP]HGfng/PyngJ) to eliminate SRR in neurons (i.e., nSRKO) or human GFAP (Gfap-CreERT2 mice to eliminate SRR in astrocytes, i.e., aSRKO). Cre recombinase was induced between 2 and 4 weeks prior to experimentation through i.p. injections of $120 \mathrm{mg} / \mathrm{kg}$ TAM (Sigma-Aldrich) at a volume of $2 \mathrm{ml} / \mathrm{kg}$ for 10 consecutive days. TAM was dissolved in a vehicle of $90 \%$ sunflower oil and $10 \%$ ethanol. All animals received TAM treatment at least 2 weeks prior to any experimental procedure unless otherwise stated, including control $\mathrm{Sr}^{\mathrm{f} / \mathrm{fl}}$ 
mice without Cre-recombinase (cSR). All mice were maintained on a C57BL/6 background, group-housed under a 12-hour light/12-hour dark cycle, and given free access to food and water.

CCI injury and perfusion. All surgical procedures were performed using an i.p. injection of ketamine-xylazine cocktail $(100 \mathrm{mg} / \mathrm{kg} / 10$ $\mathrm{mg} / \mathrm{kg}$, respectively) to anesthetize the mice, and animals were maintained on a heating pad for the duration of the procedure. A small skin incision was made to expose the skull, and mice were placed in a stereotaxic frame to provide accurate coordinates for CCI injury. Mice were subjected to a moderate CCI injury at a velocity of $4 \mathrm{~m} / \mathrm{s}, 0.50$ $\mathrm{mm}$ depth, and $150 \mathrm{~ms}$ duration using an eCCI-6.0 device (Custom Design \& Fabrication), where a hemispherical (rounded) 1.3-mm tip piston was placed over the right parieto-temporal cortex. Coordinates of the injury epicenter were $-2.0 \mathrm{~mm}$ from bregma and lateral -2.5 $\mathrm{mm}$, and a 5-mm craniotomy was performed using a small drill bit without disturbing the dura tissue layer. Skin was closed using 5-0 Vicryl synthetic absorbent sutures; mice were placed on a heating pad and monitored bidaily for 1 week after injury. At the time of harvesting, mice were intracardially perfused with $0.1 \mathrm{M}$ phosphate buffer solution (PBS) (pH 7.4) followed by either $4 \%$ paraformaldehyde (PFA) in $0.1 \mathrm{M}$ phosphate buffer (PB) ( $\mathrm{pH}$ 7.4) for immunohistochemical analysis or a glutaraldehyde-PFA fixing solution (3\% glutaraldehyde, $1 \%$ PFA, $0.2 \%$ sodium metabisulfite, and heparin).

Immunohistochemistry. Immunohistochemistry was performed as previously described (12). Briefly, 20- $\mu \mathrm{m}$ free-floating brain sections were treated with Schiff's Reagent (Sigma-Aldrich), followed by washing with $0.1 \mathrm{M}$ hydrochloric acid containing $0.5 \%$ sodium metabisulfite for 10 minutes. Sections were then washed 4 times with $0.2 \%$ sodium metabisulfite and $1.0 \%$ sodium borohydride, followed by blocking solution. Immunostaining with rabbit anti-SRR antibody (1:200, Santa Cruz Biotechnology Inc.) was performed by diluting primary antibodies in blocking solution. For astrocyte colocalization, sections were incubated with anti-GFAP antibody (1:2000, BD Biosciences). Sections were incubated with biotinylated secondary antibody and later incubated in the dark with streptavidin Alexa Fluor 488 and species-appropriate secondary Alexa Fluor $647 \operatorname{IgG}(\mathrm{H}+\mathrm{L})$.

The rabbit anti-D-serine antibody (Abcam; ab6472) was used at a dilution of 1:30,000 and incubated with $5 \mathrm{mM}$ (final concentration) L-serine-BSA blocking conjugate for 2 nights at $4^{\circ} \mathrm{C}$. Sections were then washed and incubated for 60 minutes at room temperature with the ImmPRESS Polymerized Reporter Enzyme Staining System (anti-rabbit IgG peroxidase; Vector Laboratories; RRID: AB_2336529). Sections were developed using the ImmPACT DAB Peroxidase Substrate Kit (Vector Laboratories; RRID: AB_2336520) according to the manufacturer's instructions.

CLARITY. After mice were PFA perfusion fixed, brains were incubated in a hydrogel solution (HS) for 24 hours (57). The HS allows for the removal of lipids while preserving the tissues' fine structures and also property of being permeable to light. HS consisted of $40 \%$ acrylamide (Bio-Rad), 2\% Bis (Bio-Rad), and 0.25\% VA-044 Initiator (WAKO) dissolved in 0.1 M PBS. For polymerization, brains were degassed with nitrogen and placed in a shaking incubator at $37^{\circ} \mathrm{C}$ for 3 hours. Tissue was dissected from the remaining HS and washed several times in PBS to remove any excess polymer. Sections were cut coronally at $250 \mu \mathrm{m}$ in chilled PBS, using a vibrating microtome (Leica Biosystems). Over 4 days, sections were passively cleared by incubating them in $8 \% \mathrm{SDS}$ at $37^{\circ} \mathrm{C}(58)$. For staining, tissue was incubated in primary antibodies for 3 days, washed in PBS-T (1\% Tween-20 in 0.1 M PBS), and then incubated in secondary antibodies for 3 days at room temperature. The antibodies used were polyclonal rabbit anti-GFAP (1:2000; DAKO North America, Fisher Scientific) and anti-rabbit IgG (1:1000, Thermo Fisher Scientific). Sections were washed in PBS-T and imaged on an Olympus BX50 microscope (Olympus Corp.).

Astrocyte isolation. Ipsilateral hippocampi from sham-treated and CCI-injured mice were collected, and cells were dissociated up to 400 mg tissue per sample following instructions for the Neural Tissue Dissociation Kit-Postnatal Neurons (130-094-802, Miltenyi Biotec). Hippocampi were then placed in Dulbecco's phosphate-buffered saline and cut into small pieces using a scalpel. After transferring to a $15-\mathrm{ml}$ tube, $1,960 \mu \mathrm{l}$ of enzyme mix 1 (50 $\mu \mathrm{l}$ enzyme P or $200 \mu \mathrm{l}$ enzyme T and $1910 \mu \mathrm{l}$ or $1760 \mu \mathrm{l}$ buffer $\mathrm{X}$, respectively) was added to the tube and it was gently agitated and incubated for 15 minutes at $37^{\circ} \mathrm{C}$ under slow continuous rotation. Then $30 \mu$ l of enzyme mix 2 (1:2 enzyme A to buffer Y) was added, and tissue was dissociated using a wide-tipped, fire-polished Pasteur pipette and incubated for 10 minutes at $37^{\circ} \mathrm{C}$ under slow continuous rotation. An additional $15 \mu \mathrm{l}$ of enzyme mix 2 was added, and tissue was dissociated using 2 more fire-polished Pasteur pipettes of decreasing diameter. Cell suspension was passed through a MACS SmartStrainer $70 \mu \mathrm{m}$ (130-098-462, Miltenyi Biotec), Dulbecco's phosphate-buffered saline supplemented with BSA was added, samples were centrifuged at $300 \mathrm{~g}$ for 10 minutes at room temperature, and supernatant was aspirated.

Following single cell suspension, the Anti-GLAST (ACSA-1) Microbead Kit (130-095-826, Miltenyi Biotec) was used for isolation of astrocytes. Working as fast as possible and maintaining all solutions on ice (i.e., $2-8^{\circ} \mathrm{C}$ ), cell number was determined with an automatic cell counter (Bio-Rad) for each cell suspension and volumes below were based on up to $10^{7}$ total cells. After aspiration of the supernatant, 80 $\mu \mathrm{l}$ of DPBS-BSA buffer was added followed by $20 \mu \mathrm{l}$ of Anti-GLAST (ACSA-1) Biotin Antibody (Miltenyi Biotec). Solution was mixed well, incubated at $2-8^{\circ} \mathrm{C}$ for 10 minutes, and washed by adding $2 \mathrm{ml}$ of buffer and centrifuging at $300 \mathrm{~g}$ for 10 minutes. The supernatant was aspirated and $20 \mu \mathrm{l}$ of Anti-Biotin MicroBeads (Miltenyi Biotec) were added, mixed well, and incubated for 15 minutes at $2-8^{\circ} \mathrm{C}$. Cells were washed by adding $2 \mathrm{ml}$ of buffer and centrifuged at $300 \mathrm{~g}$ for 10 minutes; supernatant was aspirated, and cells were resuspended in $500 \mu \mathrm{l}$ of buffer.

Magnetic separation was performed using LS Columns (130042-401, Miltenyi Biotec) on a MidiMACS Separator (130-042-302, Miltenyi Biotec). Cell suspension was applied to prepared columns, columns were washed with buffer $(3 \times 3 \mathrm{ml})$, and flow-through containing unlabeled cells was collected. LS Columns were removed from separator, and $5 \mathrm{ml}$ of buffer was pipetted onto the column and immediately flushed out onto a collection tube by pushing a plunger into the column. Astrocytes from GFAP-Rg reporter mice were magnetically sorted, and the purity of the positive (i.e., labeled) and negative (i.e., unlabeled flow-through) fractions was verified using a Beckman Coulter CytoFLEX S Flow Cytometer with CytExpert software 1.2. The resulting FCS files were analyzed using Beckman Coulter Kaluza 1.3 software.

Western blot analyses. Hippocampal ipsilateral tissues from sham-treated and CCI-injured mice were homogenized in radioimmunoprecipitationassay(RIPA) bufferwithproteaseinhibitorcocktail (1:100, P8340, Sigma-Aldrich), phosphatase inhibitor cocktail 2 (1:100, P5726, Sigma-Aldrich), and benzonase nuclease (1:1000, 70664, Millipore Corp.) and allowed to mix in a $4^{\circ} \mathrm{C}$ rocker for at least 15 minutes. 
Tissues were then centrifuged for 20 minutes at $4^{\circ} \mathrm{C}$, supernatant was recovered, and samples were diluted and standardized to protein concentrations, with 10-25 $\mu$ g of total protein per well. Protein samples were separated on an 8\%-10\% SDS-PAGE gel and transferred to a nitrocellulose membrane (Bio-Rad). Membranes were blocked with $5 \%$ milk or $5 \%$ BSA in $0.1 \mathrm{M}$ PB with $0.1 \%$ Tween-20 for 1 hour at room temperature and incubated overnight at $4^{\circ} \mathrm{C}$ with primary antibodies. The following primary antibodies were used: anti- $\beta$ tubulin (1:30000, Sigma-Aldrich), anti-GFAP (1:2000, BD Biosciences), and anti-SRR antibodies (1:800, Santa Cruz Biotechnology Inc.). Membranes were incubated for 1 hour at room temperature with HRP-conjugated secondary antibodies (Jackson Immunoresearch Laboratories; 1:20000, 1:5000, and 1:800 for $\beta$-tubulin [catalog T-8535], GFAP [catalog 556327], and SRR [catalog sc-5751], respectively). Bands were visualized using SuperSignal substrate (Thermo Fisher Scientific). Densitometric analysis was performed using Image (NIH). Protein measurements were standardized to $\beta$-tubulin and normalized to average WT or control sham signals.

Ventricular infusion of D-serine. Alzet pumps (1007D, Alzet) were preloaded to deliver $840 \mu \mathrm{g} / \mathrm{kg} / \mathrm{d}$ of $\mathrm{D}$-serine or vehicle for 7 days after implantation. Loaded pumps were connected to a brain infusion catheter and placed in $0.1 \mathrm{M} \mathrm{PB}$ at $37^{\circ} \mathrm{C}$ overnight (27). Immediately after CCI, the infusion device was lowered using a stereotactic holder until the tip of the cannula reached the lateral ventricle $(-2.0 \mathrm{~mm}$ from dura). The infusion device was secured to the skull without disturbing the injury using Loctite 454 Prism Gel (Henkel Corp.). The Alzet pump was then secured by placing in a small s.c. pocket in the back of the neck, and the incision was closed with a suture. Mice were housed separately in clean heated cages and allowed to recover.

Chemiluminescent assay. The ipsilateral hippocampi of anesthetized mice was dissected and immediately homogenized similarly to in the Western blot protocol. D-serine was measured by a chemiluminescent assay as previously described $(16,27)$. Briefly, a $10 \mu \mathrm{l}$ sample was mixed with $100 \mu$ l of medium containing $100 \mathrm{mM}$ Tris- $\mathrm{HCl}, \mathrm{pH}$ 8.8, 20 units $/ \mathrm{ml}$ peroxidase, and $8 \mu \mathrm{M}$ luminol. To decrease background signal of luminol, $10 \mu \mathrm{l}$ DAO (75 units $/ \mathrm{ml}$ ) was added to the sample after a 15-minute delay. A FLUOstar Omega Microplate Reader (BMG Labtech) was used to record chemiluminescence kinetics at room temperature. The linear range was 50-1,000 pmol of D-serine in the samples, and the detection limit was 30 pmol. The concentration of D-serine in each sample was compared with a standard curve and normalized to control shams.

HPLC. Amino acids were derivatized with o-phthaldialdehyde (Alfa Aesar) and $N$-tert-butyloxycarbonyl-L-cysteine (Novabiochem) as previously described $(18,59)$. Derivatized samples were resolved by a C18 column (Alltima HP C18, $3 \mathrm{~lm}$ pore, Grace Discovery Sciences) and analyzed by fluorometric detection. The HPLC system consisted of an SCL-10A controller, 2 LC-10AT VP pumps, an SIL-10AD auto injector, a DGU-20A5 degasser, and a RF-551 fluorescence monitor (all instruments by Shimadzu Corp.). The separation was accomplished by application of a binary gradient of $25 \mathrm{mM}$ sodium acetate (pH 6.5) and acetonitrile. The gradient progressed from $10 \%$ to $20 \%$ acetonitrile during a 20-minute period at a flow rate of $1.0 \mathrm{ml} / \mathrm{min}$. The column was allowed to reequilibrate to starting concentrations after each run. Amino acid concentrations were calculated using the internal standard as a comparison to standard samples run at the beginning of each session.
Quantitative real-time PCR ( $q R T-P C R)$. Hippocampal tissues from sham-treated and CCI-injured WT and aSRKO mice were prepared for qRT-PCR analysis using Direct-zol RNA Mini-Prep Kit (R2070, Zymo Research) and real time (RT) reactions using the Omniscript RT Kit (205113, QIAGEN) for RNA extraction, purification, and cDNA synthesis according to the manufacturer's instructions. The absence of reverse transcriptase (i.e., no RT) was used as a negative control. Samples were run on a QuantStudio 3 Thermocycler (Applied Biosystems) using TaqMan assays for Srr (Mm01246014_m1, Applied Biosystems), DAO (Mm00438378_m1, Applied Biosystems), or AOC-1 (Mm01328574_g1, Applied Biosystems). Glyceraldehyde 3-phosphate dehydrogenase (Gapdh, Mm99999915_g1, Applied Biosystems) was used as an endogenous control. $\Delta$ Ct was calculated by subtracting the corresponding GAPDH Ct from each sample $\mathrm{Ct}$, and $\Delta \Delta \mathrm{Ct}$ was calculated by subtracting the average $\Delta \mathrm{Ct}$ of calibrator samples from the $\Delta \mathrm{Ct}$ of experimental samples. Data were presented as $2^{-\Delta \Delta \mathrm{Ct}}$ expression. Samples were run in triplicate.

Spontaneous hippocampal oscillations ( $\theta$ analysis). Mice were anesthetized with urethane $(1.5 \mathrm{~g} / \mathrm{kg})$ and placed on a stereotaxic frame. A $25-\mu \mathrm{m}$ concentric platinum-iridium bipolar stimulation electrode and a platinum-iridium recording electrode were inserted through a craniotomy site with the following coordinates from bregma: $-2.0 \mathrm{~mm}$; lateral:-2.0 mm; depth: $1.4 \mathrm{~mm}$ (stimulation electrode) and bregma: -1.8 $\mathrm{mm}$; lateral: $-1.4 \mathrm{~mm}$; depth: -1 to $-1.5 \mathrm{~mm}$ (recording electrode). These coordinates placed the stimulation electrode in the Schaffer collaterals and the recording electrode in the CA1 stratum radiatum. To further verify the position of the recording electrode, it was advanced along the dorso-ventral axis while stimulating and observing stereotypical changes in fEPSP. After establishing a stable baseline, but before commencing LTP protocol, spontaneous hippocampal EEG was recorded sampling at $20 \mathrm{kHz}$ and analyzed with LabChart software. A fast Fourier transform (FFT) was applied to EEG recordings for power spectrum analyses. To compare mean power per frequency, we averaged the instantaneous power over a 10-minute period without stimulation.

In vivo electrophysiology. Mice were anesthetized, and electrodes were implanted as detailed for spontaneous hippocampal oscillations. Field potentials were recorded and converted to digital form using an Axoclamp 2B amplifier, Axon Instruments Digidata 1322A analog-to-digital converter, and Clampex (Axon Instruments) software for data acquisition and analysis. I/O curves were generated by delivering electrical monophasic pulses to the CA3 Schaffer collaterals from a Grass S88 stimulator and S1U5 stimulus isolation unit and measuring the fEPSP at increasing stimulus intensities. Stimulus intensities were then adjusted to give fEPSP amplitude at $45 \%-55 \%$ of the maximum. A stable baseline fEPSP slope was recorded at a rate of $1 / 60 \mathrm{~s}$ for at least 30 minutes. A paired-pulse facilitation protocol was run using interpulse intervals of 20,40, 80, 160, 320 and 480 ms. LTP was induced with HFS $(2 \times 100 \mathrm{~Hz}, 1$ second trains, 20 -second intertrain interval). Post-HFS fEPSPs were recorded for at least 60 minutes. Post-tetanus fEPSP slopes were expressed as a percentage of the average fEPSP slope from the baseline recordings. After LTP protocol, tissue was electroablated and brains were sectioned to verify position of electrodes.

Fear-conditioning test. Mice were placed in a fear-conditioning apparatus $(30 \times 24 \times 21 \mathrm{~cm}$, Coulbourn Instruments $)$ with an electric grid floor (0.8-cm spacing, 4.8-mm diameter rods) for habituation for 600 seconds. At 24 hours after habituation, the training period involved placing the mouse back in the apparatus for 120 seconds followed by a 30 -second tone $(85 \mathrm{~dB}, 2 \mathrm{kHz})$ with a $0.7-\mathrm{mA}$ foot shock for the last 2 
seconds of the tone. After the shock, mice were maintained in the box for an additional 60 seconds (total duration of 210 seconds). At 24 hours after training, mice were returned to the apparatus and freezing behavior was measured for 300 seconds to assess contextual fear conditioning. Cue fear conditioning was measured 1 hour after contextual fear assessment by measuring freezing behavior for 360 seconds during which the tone $(85 \mathrm{~dB}, 2 \mathrm{kHz})$ was delivered for the last 180 seconds. The wall coloration, lighting, and scent were changed between contextual and cue assessments, and the box was cleaned with $70 \% \mathrm{EtOH}$ and an enzymatic cleaner between animals. Freezing behavior was quantified using videobased analysis software (FreezeFrame, Coulburn Instruments).

Statistics. Data were analyzed using Student's 2-tailed $t$ test for comparison of 2 experimental groups, or 1-way or 2-way ANOVA for comparison of more than 2 groups with Bonferroni's post-hoc correction. For hippocampal electrophysiological recordings, 2-way ANOVA and repeated measures ANOVA with Bonferroni's post-hoc correction were used. Data are represented as mean \pm SEM.

Study approval. All procedures were performed on male mice 2 to 4 months of age and approved by the University of Miami Institutional Animal Care and Use Committee in accordance with the NIH Guide for the Care and Use of Laboratory Animals (National Academies Press, 2011).

\section{Author contributions}

EJP and DJL designed research studies. EJP conducted experiments. EJP, SAT, ZBL, DTB, and TJS acquired data. EJP, SAT, TJS, and DJL analyzed data. JTC and DJL provided reagents and data interpretation. EJP and DJL wrote the manuscript, and SAT, ZBL, DTB, TJS, JTC provided feedback.

\section{Acknowledgments}

We thank Jennifer Brazill for critical reading of our manuscript, Maria Cepero for her experimental support, and Jose Mier for assistance with animal husbandry. We also thank Ken McCarthy for the generous donations of the human glial fibrillary acidic protein mice. This work was supported by the Miami Project to Cure Paralysis, NIH R01NS098740 (to DJL), K99MH099252 and R00MH099252 (to DTB), R01MH05190 (to JTC), F31NS089335, and the Lois Pope Life fellowship (to EJP).

Address correspondence to: Daniel J. Liebl, Professor of Neurological Surgery, The Miami Project to Cure Paralysis, The University of Miami, 1095 NW 14th Terrace, R-48, Miami, Florida 33136, USA. Phone: 305.243.7143; Email: dliebl@miami.edu.
1. Araque A, Parpura V, Sanzgiri RP, Haydon PG. Tripartite synapses: glia, the unacknowledged partner. Trends Neurosci. 1999;22(5):208-215.

2. Perea G, Navarrete M, Araque A. Tripartite synapses: astrocytes process and control synaptic information. Trends Neurosci. 2009;32(8):421-431.

3. Santello M, Calì C, Bezzi P. Gliotransmission and the tripartite synapse. Adv Exp Med Biol. 2012;970:307-331.

4. Furman JL, et al. Blockade of astrocytic calcineurin/NFAT signaling helps to normalize hippocampal synaptic function and plasticity in a rat model of traumatic brain injury. J Neurosci. 2016;36(5):1502-1515.

5. Tyzack GE, et al. Astrocyte response to motor neuron injury promotes structural synaptic plasticity via STAT3-regulated TSP-1 expression. Nat Commun. 2014;5:4294.

6. Warren KM, Reeves TM, Phillips LL. MT5-MMP, ADAM-10, and N-cadherin act in concert to facilitate synapse reorganization after traumatic brain injury. J Neurotrauma. 2012;29(10):1922-1940.

7. Chamoun R, Suki D, Gopinath SP, Goodman JC, Robertson C. Role of extracellular glutamate measured by cerebral microdialysis in severe traumatic brain injury. J Neurosurg. 2010;113(3):564-570.

8. Faden AI, Demediuk P, Panter SS, Vink R. The role of excitatory amino acids and NMDA receptors in traumatic brain injury. Science. 1989;244(4906):798-800.

9. Palmer AM, Marion DW, Botscheller ML, Swedlow PE, Styren SD, DeKosky ST. Traumatic brain injury-induced excitotoxicity assessed in a controlled cortical impact model. J Neurochem. 1993;61(6):2015-2024.

10. Hardingham GE, Bading H. Synaptic versus extrasynaptic NMDA receptor signalling: implications for neurodegenerative disorders. Nat Rev Neurosci. 2010;11(10):682-696.

11. Hardingham GE, Fukunaga Y, Bading H. Extra- synaptic NMDARs oppose synaptic NMDARs by triggering CREB shut-off and cell death pathways. Nat Neurosci. 2002;5(5):405-414.

12. Balu DT, Takagi S, Puhl MD, Benneyworth MA, Coyle JT. D-serine and serine racemase are localized to neurons in the adult mouse and human forebrain. Cell Mol Neurobiol. 2014;34(3):419-435.

13. Schell MJ, Brady RO, Molliver ME, Snyder SH. D-serine as a neuromodulator: regional and developmental localizations in rat brain glia resemble NMDA receptors. J Neurosci. 1997;17(5):1604-1615.

14. Schell MJ, Molliver ME, Snyder SH. D-serine, an endogenous synaptic modulator: localization to astrocytes and glutamate-stimulated release. Proc Natl Acad Sci U S A. 1995;92(9):3948-3952.

15. Wolosker H, Blackshaw S, Snyder SH. Serine racemase: a glial enzyme synthesizing $\mathrm{D}$-serine to regulate glutamate-N-methyl-D-aspartate neurotransmission. Proc Natl Acad Sci U S A. 1999;96(23):13409-13414.

16. Wolosker $\mathrm{H}$, et al. Purification of serine racemase: biosynthesis of the neuromodulator D-serine. Proc Natl Acad Sci U S A. 1999;96(2):721-725

17. Matsui T, Sekiguchi M, Hashimoto A, Tomita U, Nishikawa T, Wada K. Functional comparison of $\mathrm{D}$-serine and glycine in rodents: the effect on cloned NMDA receptors and the extracellular concentration. J Neurochem. 1995;65(1):454-458.

18. Benneyworth MA, Li Y, Basu AC, Bolshakov VY, Coyle JT. Cell selective conditional null mutations of serine racemase demonstrate a predominate localization in cortical glutamatergic neurons. Cell Mol Neurobiol. 2012;32(4):613-624.

19. Miya K, et al. Serine racemase is predominantly localized in neurons in mouse brain. J Comp Neurol. 2008;510(6):641-654

20. Rosenberg D, Kartvelishvily E, Shleper M, Klinker CM, Bowser MT, Wolosker H. Neuronal release of D-serine: a physiological pathway controlling extracellular D-serine concentration.
FASEB J. 2010;24(8):2951-2961.

21. Wolosker H, Balu DT, Coyle JT. The rise and fall of the d-serine-mediated gliotransmission hypothesis. Trends Neurosci. 2016;39(11):712-721.

22. Inoue R, Hashimoto K, Harai T, Mori H. NMDAand beta-amyloid1-42-induced neurotoxicity is attenuated in serine racemase knock-out mice. JNeurosci. 2008;28(53):14486-14491.

23. Katsuki H, Nonaka M, Shirakawa H, Kume T, Akaike A. Endogenous D-serine is involved in induction of neuronal death by N-methyl-D-aspartate and simulated ischemia in rat cerebrocortical slices. JPharmacol Exp Ther. 2004;311(2):836-844.

24. Mustafa AK, et al. Serine racemase deletion protects against cerebral ischemia and excitotoxicity. J Neurosci. 2010;30(4):1413-1416.

25. Papouin T, Oliet SH. Organization, control and function of extrasynaptic NMDA receptors. Philos Trans R Soc Lond, B, Biol Sci. 2014;369(1654):20130601.

26. Shleper M, Kartvelishvily E, Wolosker H. D-serine is the dominant endogenous coagonist for NMDA receptor neurotoxicity in organotypic hippocampal slices. J Neurosci. 2005;25(41):9413-9417.

27. Perez EJ, Cepero ML, Perez SU, Coyle JT, Sick TJ, Liebl DJ. EphB3 signaling propagates synaptic dysfunction in the traumatic injured brain. Neurobiol Dis. 2016;94:73-84.

28. Hablitz JJ, Langmoen IA. N-methyl-D-aspartate receptor antagonists reduce synaptic excitation in the hippocampus. J Neurosci.1986;6(1):102-106.

29. Derkach VA, Oh MC, Guire ES, Soderling TR. Regulatory mechanisms of AMPA receptors in synaptic plasticity. Nat Rev Neurosci. 2007;8(2):101-113.

30. Paoletti P, Bellone C, Zhou Q. NMDA receptor subunit diversity: impact on receptor properties, synaptic plasticity and disease. Nat Rev Neurosci. 2013;14(6):383-400.

31. Rosenberg D, et al. Neuronal D-serine and gly- 
cine release via the Asc-1 transporter regulates NMDA receptor-dependent synaptic activity. JNeurosci. 2013;33(8):3533-3544.

32. DeVito LM, et al. Serine racemase deletion disrupts memory for order and alters cortical dendritic morphology. Genes Brain Behav. 2011;10(2):210-222.

33. Kartvelishvily E, Shleper M, Balan L, Dumin E, Wolosker H. Neuron-derived D-serine release provides a novel means to activate $\mathrm{N}$-methyl-D-aspartate receptors. J Biol Chem. 2006;281(20):14151-14162.

34. Theus MH, Ricard J, Glass SJ, Travieso LG, Liebl DJ. EphrinB3 blocks EphB3 dependence receptor functions to prevent cell death following traumatic brain injury. Cell Death Dis. 2014;5:e1207.

35. Assis-Nascimento P, Umland O, Cepero ML, Liebl DJ. A flow cytometric approach to analyzing mature and progenitor endothelial cells following traumatic brain injury. J Neurosci Methods. 2016;263:57-67.

36. Obrenovitch TP, Urenjak J. Is high extracellular glutamate the key to excitotoxicity in traumatic brain injury? J Neurotrauma. 1997;14(10):677-698.

37. Albin RL, Greenamyre JT. Alternative excitotoxic hypotheses. Neurology. 1992;42(4):733-738.

38. Ikonomidou C, Turski L. Excitotoxicity and neurodegenerative diseases. Curr Opin Neurol. 1995;8(6):487-497.

39. Fedor M, Berman RF, Muizelaar JP, Lyeth BG. Hippocampal $\theta$ dysfunction after lateral fluid percussion injury. JNeurotrauma. 2010;27(9):1605-1615.

40. Paterno R, Metheny H, Xiong G, Elkind J, Cohen AS. Mild traumatic brain injury decreases broadband power in area CA1. J Neurotrauma. 2016;33(17):1645-1649.
41. McIntosh TK, et al. Traumatic brain injury in the rat: characterization of a lateral fluid-percussion model. Neuroscience. 1989;28(1):233-244.

42. Bland BH. The physiology and pharmacology of hippocampal formation theta rhythms. Prog Neurobiol. 1986;26(1):1-54.

43. Bland BH, Bland CE, MacIver MB. Median raphe stimulation-induced motor inhibition concurrent with suppression of type 1 and type 2 hippocampal theta. Hippocampus. 2016;26(3):289-300.

44. Hassanpoor H, Fallah A, Raza M. Mechanisms of hippocampal astrocytes mediation of spatial memory and theta rhythm by gliotransmitters and growth factors. Cell Biol Int. 2014;38(12):1355-1366.

45. Korotkova T, Fuchs EC, Ponomarenko A, von Engelhardt J, Monyer H. NMDA receptor ablation on parvalbumin-positive interneurons impairs hippocampal synchrony, spatial representations, and working memory. Neuron. 2010;68(3):557-569.

46. Araque A, Sanzgiri RP, Parpura V, Haydon PG. Calcium elevation in astrocytes causes an NMDA receptor-dependent increase in the frequency of miniature synaptic currents in cultured hippocampal neurons. J Neurosci. 1998;18(17):6822-6829.

47. Guo JD, Wang H, Zhang YQ, Zhao ZQ. Alterations of membrane properties and effects of D-serine on NMDA-induced current in rat anterior cingulate cortex neurons after monoarthritis. Neurosci Lett. 2005;384(3):245-249.

48. Wirkner K, et al. Modulation of NMDA receptor current in layer $\mathrm{V}$ pyramidal neurons of the rat prefrontal cortex by P2Y receptor activation. Cereb Cortex. 2007;17(3):621-631.

49. Bramlett HM, Dietrich WD. Progressive damage after brain and spinal cord injury: pathomechanisms and treatment strategies. Prog Brain Res. 2007;161:125-141.

50. Bullock R, et al. Factors affecting excitatory amino acid release following severe human head injury. J Neurosurg. 1998;89(4):507-518.

51. Nilsson P, Hillered L, Pontén U, Ungerstedt U. Changes in cortical extracellular levels of energy-related metabolites and amino acids following concussive brain injury in rats. J Cereb Blood Flow Metab. 1990;10(5):631-637.

52. Erreger K, et al. Subunit-specific agonist activity at NR2A-, NR2B-, NR2C-, and NR2D-containing $\mathrm{N}$-methyl-D-aspartate glutamate receptors. $\mathrm{Mol}$ Pharmacol. 2007;72(4):907-920.

53. Papouin T, et al. Synaptic and extrasynaptic NMDA receptors are gated by different endogenous coagonists. Cell. 2012;150(3):633-646.

54. Le Bail M, et al. Identity of the NMDA receptor coagonist is synapse specific and developmentally regulated in the hippocampus. Proc Natl Acad Sci U S A. 2015;112(2):E204-E213.

55. Traynelis SF, et al. Glutamate receptor ion channels: structure, regulation, and function. Pharmacol Rev. 2010;62(3):405-496.

56. Casper KB, Jones K, McCarthy KD. Characterization of astrocyte-specific conditional knockouts. Genesis. 2007;45(5):292-299.

57. Chung K, Deisseroth K. CLARITY for mapping the nervous system. Nat Methods. 2013;10(6):508-513.

58. Tomer R, Ye L, Hsueh B, Deisseroth K. Advanced CLARITY for rapid and high-resolution imaging of intact tissues. Nat Protoc. 2014;9(7):1682-1697.

59. Hashimoto A, et al. The presence of free D-serine in rat brain. FEBS Lett. 1992;296(1):33-36. 